\title{
Reversion Porisms in Conics
}

\section{Lorenz Halbeisen, Norbert Hungerbühler*and Marco Schiltknecht}

\author{
(Communicated by Levent Kula)
}

\begin{abstract}
We give a projective proof of the butterfly porism for cyclic quadrilaterals and present a general reversion porism for polygons with an arbitrary number of vertices on a conic. We also investigate projective properties of the porisms.
\end{abstract}

Keywords: Porisms, butterfly theorems, reversion map.

AMS Subject Classification (2020): Primary: 51M15; Secondary: 51M09.

\section{Introduction}

The theorems of Pappus and Pascal and the Scissors Theorem can be formulated as porisms in the projective plane:

Theorem 1 (Pappus). Let $A_{1}, A_{2}, \ldots, A_{6}$ be a Pappus hexagon on the lines $\ell_{1}, \ell_{2}$ with intersection points $P_{1}, P_{2}, P_{3}$ on the Pappus line $\ell$. Then there exists a Pappus hexagon $A_{1}^{\prime}, A_{2}^{\prime}, \ldots, A_{6}^{\prime}$ on $\ell_{1}, \ell_{2}$ with the same intersection points $P_{1}, P_{2}, P_{3}$ for any point $A_{1}^{\prime}$ on $\ell_{1}$ (see Figure 1 ).

The cases when $A_{1}^{\prime}$ is the intersection of $\ell_{1}$ with $\ell$ or $\ell_{2}$ are considered as degenerate situations.

Proof of Theorem 1. By the Theorem of Pappus, applied to the hexagon $A_{1}, \ldots, A_{6}$, the points $P_{1}, P_{2}, P_{3}$ are collinear. Then the Braikenridge-Maclaurin Theorem for degenerate conics (see, e.g., [2, p. 76]) applied to the points $A_{1}^{\prime}, A_{2}^{\prime}, \ldots, A_{5}^{\prime}$ and the points $P_{1}, P_{2}, P_{3}$ implies that $A_{6}^{\prime}$ lies on $\ell_{2}$.

Theorem 2 (Pascal). Let $A_{1}, A_{2}, \ldots, A_{6}$ be a Pascal hexagon on the conic $C$ with intersection points $P_{1}, P_{2}, P_{3}$ on the Pascal line $\ell$. Then there exists a Pascal hexagon $A_{1}^{\prime}, A_{2}^{\prime}, \ldots, A_{6}^{\prime}$ on $C$ with the same intersection points $P_{1}, P_{2}, P_{3}$ for any point $A_{1}^{\prime}$ on $C$ (see Figure 2).

Again, we consider the case when $A_{1}^{\prime}$ is on $\ell$ as a degenerate situation.

Proof of Theorem 2. By the Theorem of Pascal, applied to the hexagon $A_{1}, \ldots, A_{6}$, the points $P_{1}, P_{2}, P_{3}$ are collinear. Then the Braikenridge-Maclaurin Theorem for nondegenerate conics applied to the points $A_{1}^{\prime}, A_{2}^{\prime}, \ldots, A_{5}^{\prime}$ and the points $P_{1}, P_{2}, P_{3}$ implies that $A_{6}^{\prime}$ lies on $C$.

Theorem 3 (Scissors Theorem). Let $A_{1}, A_{2}, A_{3}, A_{4}$ be a Scissors quadrilateral on the lines $\ell_{1}, \ell_{2}$ with intersection points $P_{1}, P_{2}, P_{3}, P_{4}$ on a line $\ell$. Then there exists a Scissors quadrilateral $A_{1}^{\prime}, A_{2}^{\prime}, A_{3}^{\prime}, A_{4}^{\prime}$ on $\ell_{1}, \ell_{2}$ with the same intersection points $P_{1}, P_{2}, P_{3}, P_{4}$ for any point $A_{1}^{\prime}$ on $\ell_{1}$ (see Figure 3 ). 


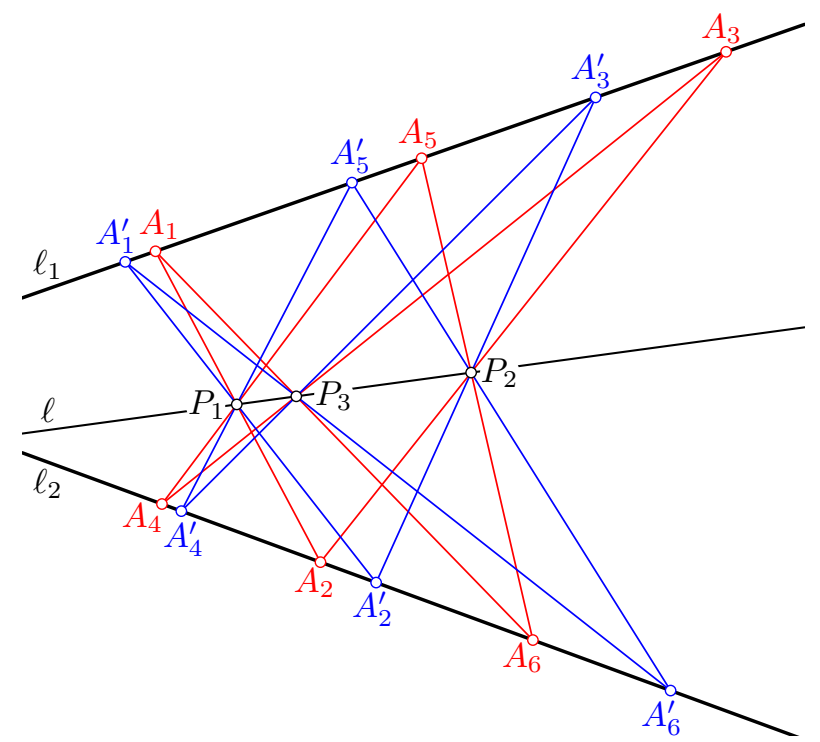

Figure 1. Pappus hexagons $A_{1}, A_{2}, \ldots, A_{6}$ and $A_{1}^{\prime}, A_{2}^{\prime}, \ldots, A_{6}^{\prime}$.

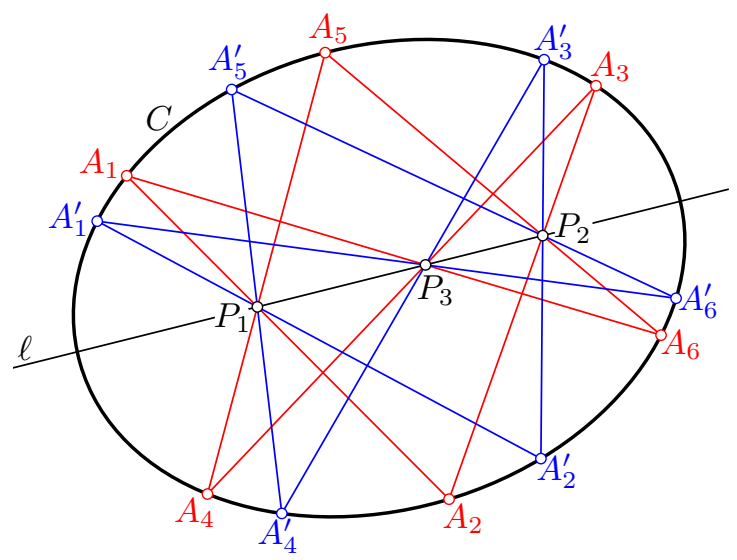

Figure 2. Pascal hexagons $A_{1}, A_{2}, \ldots, A_{6}$ and $A_{1}^{\prime}, A_{2}^{\prime}, \ldots, A_{6}^{\prime}$.

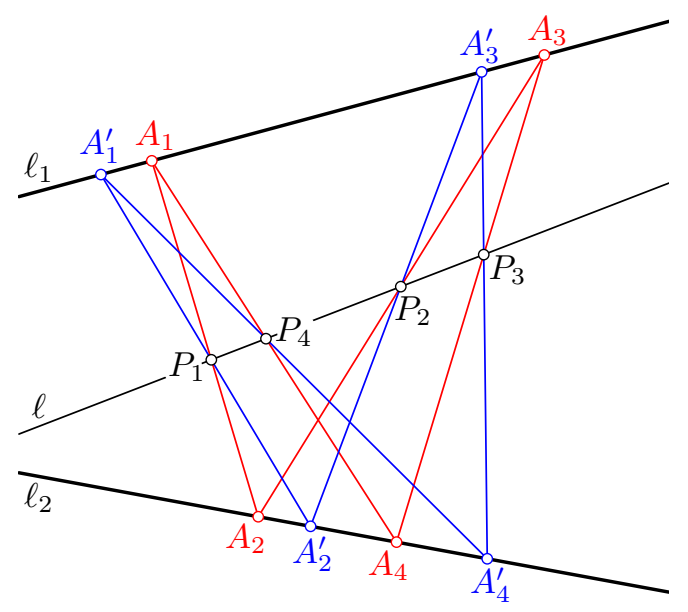

Figure 3. Scissors quadrilaterals $A_{1}, A_{2}, \ldots, A_{4}$ and $A_{1}^{\prime}, A_{2}^{\prime}, \ldots, A_{4}^{\prime}$. 
Also here, the cases when $A_{1}^{\prime}$ is the intersection of $\ell_{1}$ with $\ell$ or $\ell_{2}$ are included in the theorem as degenerate situations.

Proof of Theorem 3. In the real affine plane obtained by removing $\ell$ from the projective plane the lines $A_{i} A_{i+1}$ and $A_{i}^{\prime} A_{i+1}^{\prime}$ (with cyclically read indices) are parallel. Then the quadrilateral $A_{1}^{\prime}, A_{2}^{\prime}, A_{3}^{\prime}, A_{4}^{\prime}$ is obtained from $A_{1}, A_{2}, A_{3}, A_{4}$ by a translation (if $\ell_{1}, \ell_{2}$ and $\ell$ are concurrent) or by a homothetic transformation (if $\ell_{1}, \ell_{2}$ and $\ell$ are not concurrent).

Formally, one obtains the Pascal Porism 2 from the Pappus Porism 1 by replacing the degenerate conic $\ell_{1} \cup \ell_{2}$ by the nondegenerate conic $C$. Surprisingly, one obtains another porism by the same process when we replace the degenerate conic $\ell_{1} \cup \ell_{2}$ in the Scissors Porism 3 by a nondegenerate conic $C$ :

Theorem 4 (Butterfly Theorem, Jones [4], Kocik [6]). Let $A_{1}, A_{2}, A_{3}, A_{4}$ be a quadrilateral on the conic $C$ with intersection points $P_{1}, P_{2}, P_{3}, P_{4}$ on a line $\ell$. Then there exists a quadrilateral $A_{1}^{\prime}, A_{2}^{\prime}, A_{3}^{\prime}, A_{4}^{\prime}$ on $C$ with the same intersection points $P_{1}, P_{2}, P_{3}, P_{4}$ for any point $A_{1}^{\prime}$ on $C$ (see Figure 4 ).

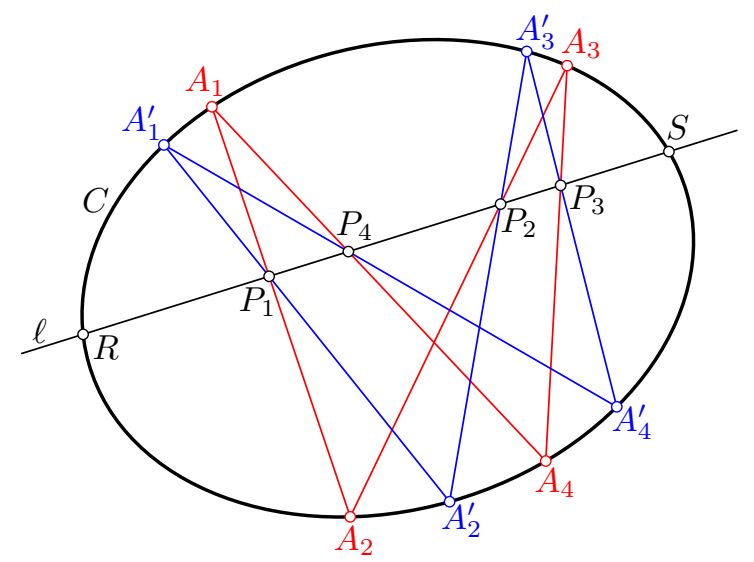

Figure 4. Butterfly quadrilaterals $A_{1}, A_{2}, \ldots, A_{4}$ and $A_{1}^{\prime}, A_{2}^{\prime}, \ldots, A_{4}^{\prime}$.

The theorem of Jones and Kocik generalizes the well known classical Butterfly Theorem (see, e.g., [5], [7], [8], and the many variants in [1]). Kocik proved the theorem in the setting of the complex plane using Möbius transformations, thus excluding the case that one of the points $P_{i}$ lies on the ideal line. He also proved a corresponding theorem for polygons $A_{1}, A_{2}, \ldots, A_{2 n}, n \geq 2$, if the points $P_{1}, P_{2}, \ldots, P_{2 n}$ are collinear (see [6]). Izmestiev quantified in [3] Kocik's Theorem using cross-ratios by proving, that

$$
\operatorname{cr}\left(R, S ; P_{1}, P_{4}\right)=\operatorname{cr}\left(R, S ; P_{2}, P_{3}\right),
$$

is a necessary and sufficient condition for the closing of the quadrilaterals. Here $\ell$ intersects $C$ in the points $R, S$ (see Figure 4). (The same relation holds for the Scissors Porism 3 when $R$ and $S$ are the intersections of $\ell$ with $\ell_{1}$ and $\ell_{2}$, respectively.) Izmestiev gave a similar characterisation if $\ell$ is tangent to $C$ or does not intersect $C$ (see [3]). He also proved that the closing condition is satisfied for a polygon $A_{1}, A_{2}, \ldots, A_{n}, n \geq 5$, in a circle $C$ if the points $P_{1}, P_{2}, \ldots, P_{n}$ form a right-angled polygon when the interior of $C$ is viewed as the Cayley-Klein model of the hyperbolic plane. This is a sufficient, but not a necessary closing condition. In particular, it only applies to points $P_{i}$ inside $C$.

In Section 2 we will prove the Butterfly Porism 4 in a purely projective manner using projective maps instead of Möbius transformations, thus closing the gap in the previous proofs. In Section 3 we will give a necessary and sufficient closing condition for the points $P_{1}, \ldots, P_{n}, n \geq 3$ : It will turn out that the condition is a consequence of Pascal's Theorem. In particular, given an $n$-gon $A_{1}, A_{2}, \ldots, A_{n}$ inscribed in a conic $C$ with points $P_{1}, \ldots, P_{n-2}$ such that $P_{i}$ lies on the line $A_{i} A_{i+1}$, there are unique points $P_{n-1}$ on $A_{n-1} A_{n}$ and $P_{n}$ on $A_{n} A_{1}$ such that the closing condition is satisfied. I.e., there is an $n$-gon $A_{1}^{\prime} A_{2}^{\prime} \ldots A_{n}^{\prime}$ on $C$ with sides running successively through the points $P_{i}$ for any starting point $A_{1}^{\prime}$ on $C$. The points $P_{n-1}$ and $P_{n}$ can easily be constructed by ruler alone. 


\section{The Butterfly Porism}

Let $C$ be a nondegenerate conic in the real projective plane $\mathbb{R P}^{2}$. For a point $P \notin C$, the reversion map $\varphi_{P}: C \rightarrow C, X \mapsto \varphi_{P}(X)$, is defined by the requirement that $X, P, \varphi_{P}(X)$ are collinear, and that $X \neq \varphi_{P}(X)$ unless $X P$ is a tangent of $C$ (see Figure 5). If convenient, we may always assume without loss of generality that the conic $C$ is given by

$$
C:\langle X, X\rangle=0
$$

in projective coordinates $X=\left(x_{1}, x_{2}, x_{3}\right)^{\top}$, where $\langle X, Y\rangle=x_{1} y_{1}+x_{2} y_{2}-x_{3} y_{3}$ denotes the Minkowski product. In the Euclidean plane $\left\{\left(x_{1}, x_{2}, 1\right) \mid\left(x_{1}, x_{2}\right) \in \mathbb{R}^{2}\right\}$, embedded in the projective plane $\mathbb{R P}^{2}, C$ is the unit circle.

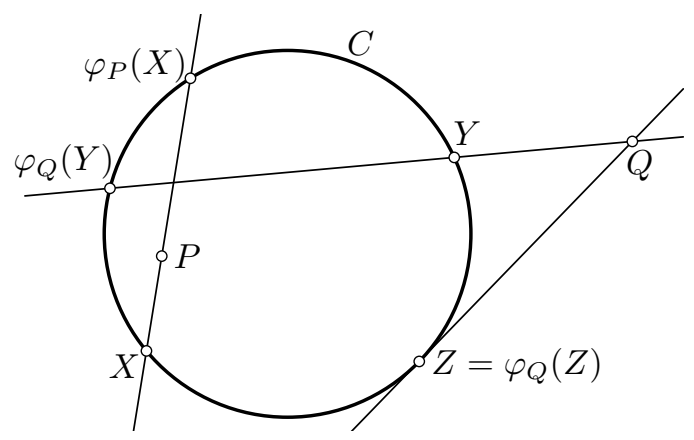

Figure 5. The reversion map on $C$.

Observe that $\varphi_{P}$ has a unique continuation as a projective map $\mathbb{R} \mathrm{P}^{2} \rightarrow \mathbb{R P}^{2}$ which we also denote by $\varphi_{P}$ and which is given by

$$
X \mapsto \varphi_{P}(X)=M_{P} X
$$

where

$$
M_{P}=\left(\begin{array}{ccc}
-p_{1}^{2}+p_{2}^{2}-p_{3}^{2} & -2 p_{1} p_{2} & 2 p_{1} p_{3} \\
-2 p_{1} p_{2} & p_{1}^{2}-p_{2}^{2}-p_{3}^{2} & 2 p_{2} p_{3} \\
-2 p_{1} p_{3} & -2 p_{2} p_{3} & p_{1}^{2}+p_{2}^{2}+p_{3}^{2}
\end{array}\right) .
$$

Note that the polar line of $P$ is a fixed point line of $\varphi_{P}$ and the bundle of lines through $P$ are fixed lines of $\varphi_{P}$. Moreover, $\varphi_{P}$ is an involution.

Lemma 5. If $U, V, W \in \mathbb{R} \mathrm{P}^{2} \backslash C$ are collinear, then there is a unique point $X \in \mathbb{R} \mathrm{P}^{2} \backslash C$ on the same line as $U, V, W$ such that $\varphi_{X} \circ \varphi_{W} \circ \varphi_{V} \circ \varphi_{U}=$ id, i.e., the identity.

Proof. $W$ is given by $W=a U+b V$ for some $a, b \in \mathbb{R}$. Observe that

$$
\langle U, U\rangle \neq 0, \quad\langle V, V\rangle \neq 0, \quad\langle W, W\rangle \neq 0,
$$

since $U, V, W \notin C$. Set $X:=(2 a\langle U, V\rangle+b\langle V, V\rangle) U-a\langle U, U\rangle V$. Then a short calculation shows that

$$
\langle X, X\rangle=\langle U, U\rangle\langle V, V\rangle\langle W, W\rangle \neq 0
$$

and hence $X \notin C$. It is then elementary to check that $M_{X}=M_{W} M_{V} M_{U}$.

The Butterfly Porism 4 follows immediately from Lemma 5 with $P_{1}=U, P_{2}=V, P_{3}=W$ : Indeed, Lemma 5 guarantees the existence of a point $X$ on the line $\ell$ such that $A_{2}=\varphi_{P_{1}}\left(A_{1}\right), A_{3}=\varphi_{P_{2}}\left(A_{2}\right), A_{4}=\varphi_{P_{3}}\left(A_{3}\right)$, and $A_{1}=\varphi_{X}\left(A_{4}\right)$. Thus, $X=P_{4}$, and because $\varphi_{P_{4}} \circ \ldots \circ \varphi_{P_{1}}=\mathrm{id}$, the path closes for any starting point $A_{1}^{\prime}$ on $C$.

Is it possible that a closing theorem for quadrilaterals in a conic $C$ holds if the points $P_{1}, \ldots, P_{4}$ are not collinear? The answer is no:

Theorem 6. Let $A_{i}, B_{i}, C_{i}, D_{i}$ for $i=1,2,3$ be three quadrilaterals with vertices on a conic $C$ with points $P_{1}$ on $A_{i} B_{i}$, $P_{2}$ on $B_{i} C_{i}, P_{3}$ on $C_{i} D_{i}, P_{4}$ on $D_{i} A_{i}$, for $i=1,2,3$. Assume that the four points $P_{j}$ are not on $C$. Then, $P_{1}, P_{2}, P_{3}, P_{4}$ are collinear. 
Proof. Consider the following four hexagons

$$
\begin{aligned}
& A_{1} B_{1} D_{2} A_{2} B_{2} D_{1}, \\
& A_{1} B_{1} D_{3} A_{3} B_{3} D_{1}, \\
& C_{1} B_{1} D_{2} C_{3} B_{2} D_{1}, \\
& C_{1} B_{1} D_{3} C_{3} B_{3} D_{1} .
\end{aligned}
$$

Let $X$ be the intersection of the lines $B_{1} D_{2}$ and $B_{2} D_{1}$ and $Y$ the intersection of the lines $B_{1} D_{3}$ and $B_{3} D_{1}$. Then, by Pascal's Theorem applied to the four hexagons above, we have:

$$
\begin{aligned}
& \text { by (2): the points } P_{1}, P_{4}, X \text { are collinear } \\
& \text { by (3): the points } P_{1}, P_{4}, Y \text { are collinear } \\
& \text { by (4): the points } P_{2}, P_{3}, X \text { are collinear } \\
& \text { by (5): the points } P_{2}, P_{3}, Y \text { are collinear }
\end{aligned}
$$

Hence, $P_{1}, P_{2}, P_{3}, P_{4}$ are collinear.

\section{General reversion porisms in conics}

Motivated by the previous section we define:

Definition 7. Let $C$ be a conic in the projective plane $\mathbb{R P}^{2}$. The points $P_{1}, \ldots, P_{n}$ in $\mathbb{R} \mathrm{P}^{2} \backslash C$ are said to satisfy the closing property (in this order) with respect to $C$, if $\varphi_{P_{n}} \circ \ldots \circ \varphi_{P_{1}}=$ id, i.e., the identity.

Then we have the following:

Lemma 8. Let $C$ be a nondegenerate conic in the real projective plane $\mathbb{R} \mathrm{P}^{2}$ and $P_{1}, \ldots, P_{n}$ be points in $\mathbb{R P}^{2} \backslash C$. Assume that there are three different $n$-gons $A_{1}^{j} A_{2}^{j} \ldots A_{n}^{j}, j=1,2,3$, inscribed in $C$ such that $P_{i}$ lies on $A_{i} A_{i+1}$ for $i=1,2, \ldots, n$ (with cyclically read indices). Then $P_{1}, \ldots, P_{n}$ have the closing property with respect to $C$. In particluar there is a closed $n$-gon starting in any point $A_{1} \in C$ whose sides run successively through the points $P_{i}$.

Proof. $A_{1}^{1}, A_{1}^{2}$ and $A_{1}^{3}$ are three different fixed points of the map $\varphi_{P_{n}} \circ \ldots \circ \varphi_{P_{1}}$. Thus, the claim follows directly from the fact that the group of projective maps which keep the set $C$ fixed acts sharply 3-transitively on $C$.

Before we can turn to the main theorem, we need to state the following:

Proposition 9. Let $C$ be a nondegenerate conic and $U \neq V$ be points in $\mathbb{R} \mathrm{P}^{2} \backslash C$. Then $\varphi=\varphi_{V} \circ \varphi_{U}$ has the line $\ell=U V$ as a fixed line and its pole $P$ with respect to $C$ as a fixed point. Moreover we have:

(a) If $\ell$ intersects $C$ in two points $R, S$ then $R, S$ are fixed points of $\varphi$ and the tangents in $R, S$ are fixed lines. Besides $P$ and $\ell$ there are no other fixed points and fixed lines.

(b) If $\ell$ is tangent to $C$ or if $\ell$ misses $C$, then $P$ is the only fixed point and $\ell$ the only fixed line.

In particular, the line $\ell$ on which $U$ and $V$ sit is determined by the map $\varphi$.

Proof. The fixed points of $\varphi$ are the real eigenvectors of $M=M_{V} M_{U}$ and the fixed lines are the real eigenvectors of $M^{-\top}$ (the inverse transposed of $M$ ). In case (a) it is geometrically clear, that $R, S, P$ are fixed points and that $\ell$ and the tangents in $R, S$ are fixed lines. Since we have at most three real eigenvectors, there are no other fixed points or lines. In case (b) a short calculation shows, that $P$ is a triple eigenvector of $M$, and $\ell$ a triple eigenvector of $M^{-\top}$ if $\ell$ is tangent to $C$. If $\ell$ misses $C$, there is only one real eigenvalue of $M$, namely $P$, and only one real eigenvalue of $M^{-\top}$, namely $\ell$.

Now we are ready for the main theorem.

Theorem 10. Let $C$ be a nondegenerate conic and $P_{1}, \ldots, P_{n-2}$ be given points in $\mathbb{R P}^{2} \backslash C$. Then the following is true: 
- If $P_{1}, \ldots, P_{n-2}$ have the closing property with respect to $C$, then $P_{1}, \ldots, P_{n-2}, P_{n-1}, P_{n}$ have the closing property if and only if $P_{n-1}=P_{n}$ is an arbitrary point in $\mathbb{R} \mathrm{P}^{2} \backslash C$.

- If $P_{1}, \ldots, P_{n-2}$ do not have the closing property with respect to $C$, then either $\varphi=\varphi_{P_{n-2}} \circ \ldots \circ \varphi_{P_{1}}$ has a unique fixed line $\ell$ which intersects $C$ in two points, or $\varphi$ has only one fixed line $\ell$ at all. For an arbitrary point $P_{n-1} \in \ell \backslash C$ there is a unique point $P_{n} \in \ell \backslash C$ such that $P_{1}, \ldots, P_{n-2}, P_{n-1}, P_{n}$ have the closing property. No other choice for $P_{n-1}$ and $P_{n}$ is possible.

Proof. The first case is trivial, since $\varphi_{P_{n}} \circ \varphi_{P_{n-1}} \circ \ldots \circ \varphi_{P_{1}}=\varphi_{P_{n}} \circ \varphi_{P_{n-1}}=$ id implies that $\varphi_{P_{n-1}}=\varphi_{P_{n}}$ and hence $P_{n-1}=P_{n}$.

In the second case we assume that $\varphi=\varphi_{P_{n-2}} \circ \ldots \circ \varphi_{P_{1}} \neq \mathrm{id}$. We start by showing that $P_{n-1}$ and $P_{n}$ exist as specified in the theorem. In this case, $\varphi$ has at most two fixed points on $C$. So, let us first choose an arbitrary point $A_{1} \neq \varphi\left(A_{1}\right)$. Then, we can choose two different points $A_{1}^{\prime}, A_{1}^{\prime \prime} \notin\left\{\varphi^{-1}\left(A_{1}\right), \varphi\left(A_{1}\right)\right\}$ such that $A_{1}^{\prime} \neq \varphi\left(A_{1}^{\prime}\right)$ and $A_{1}^{\prime \prime} \neq \varphi\left(A_{1}^{\prime \prime}\right)$. This defines the polygonal chains

$$
\begin{aligned}
& A_{1}, A_{2}=\varphi_{P_{1}}\left(A_{1}\right), A_{3}=\varphi_{P_{2}}\left(A_{2}\right), \ldots, A_{n-1}=\varphi_{P_{n-2}}\left(A_{n-2}\right)=\varphi\left(A_{1}\right), \\
& A_{1}^{\prime}, A_{2}^{\prime}=\varphi_{P_{1}}\left(A_{1}^{\prime}\right), A_{3}^{\prime}=\varphi_{P_{2}}\left(A_{2}^{\prime}\right), \ldots, A_{n-1}^{\prime}=\varphi_{P_{n-2}}\left(A_{n-2}^{\prime}\right)=\varphi\left(A_{1}^{\prime}\right), \\
& A_{1}^{\prime \prime}, A_{2}^{\prime \prime}=\varphi_{P_{1}}\left(A_{1}^{\prime \prime}\right), A_{3}^{\prime \prime}=\varphi_{P_{2}}\left(A_{2}^{\prime \prime}\right), \ldots, A_{n-1}^{\prime \prime}=\varphi_{P_{n-2}}\left(A_{n-2}^{\prime \prime}\right)=\varphi\left(A_{1}^{\prime \prime}\right) .
\end{aligned}
$$

Then the intersection $X$ of the lines $A_{1} A_{n-1}^{\prime}$ with the line $A_{1}^{\prime} A_{n-1}$ and the intersection $Y$ of the lines $A_{1} A_{n-1}^{\prime \prime}$ with the line $A_{1}^{\prime \prime} A_{n-1}$ are different and define a line $\ell$ (see Figure 6). Choose a point $P_{n-1}$ on $\ell$ such that the line $A_{n-1} P_{n-1}$ intersects $C$ in a point $A_{n}$. The line $A_{n} A_{1}$ then intersects $\ell$ in a point $P_{n}$. Hence, $A_{n}=\varphi_{P_{n-1}}\left(A_{n-1}\right)$ and $A_{1}=\varphi_{P_{n}}\left(A_{n}\right)$. Now we consider the intersection $A_{n}^{\prime}$ of the lines $A_{n-1}^{\prime} P_{n-1}$ and $A_{1}^{\prime} P_{n}$ and the intersection

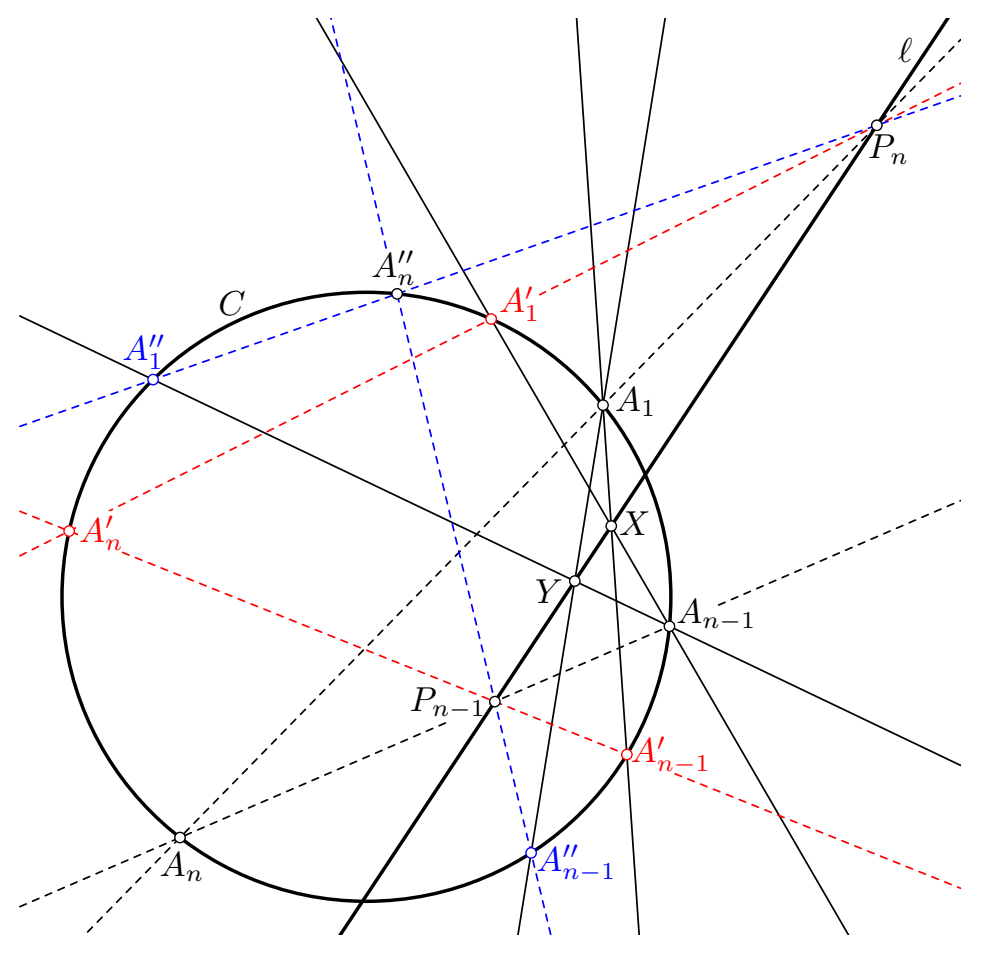

Figure 6. Proof of Theorem 10

$A_{n}^{\prime \prime}$ of the lines $A_{n-1}^{\prime \prime} P_{n-1}$ and $A_{1}^{\prime \prime} P_{n}$. By the Braikenridge-Maclaurin Theorem applied to the hexagon $H_{1}=$ $A_{1} A_{n-1}^{\prime} A_{n}^{\prime} A_{1}^{\prime} A_{n-1} A_{n}$ it follows that $A_{n}^{\prime} \in C$. Similarly, by considering the hexagon $H_{2}=A_{1} A_{n-1}^{\prime \prime} A_{n}^{\prime \prime} A_{1}^{\prime \prime} A_{n-1} A_{n}$ it follows that $A_{n}^{\prime \prime} \in C$. It follows that the map $\varphi_{P_{n}} \circ \ldots \circ \varphi_{P_{1}}$ has the fixed points $A_{1}, A_{1}^{\prime}$ and $A_{1}^{\prime \prime}$ on $C$ and is hence the identity. In particular, we see that

$$
\varphi=\varphi_{P_{n-2}} \circ \ldots \circ \varphi_{P_{1}}=\varphi_{P_{n-1}} \circ \varphi_{P_{n}} .
$$

Hence, by Proposition 9, the line $\ell$ on which $P_{n-1}$ and $P_{n}$ sit is determined by the points $P_{1}, \ldots, P_{n-2}$, and clearly, $P_{n}$ is determined as soon as $P_{n-1}$ is chosen on $\ell \backslash C$. 


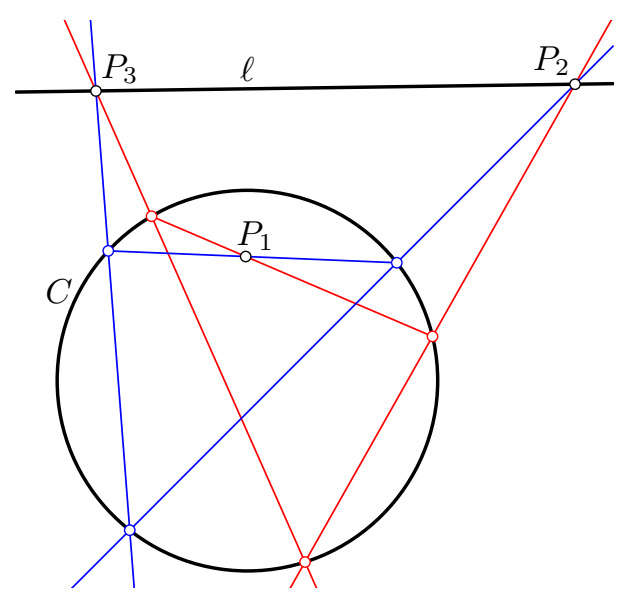

Figure 7. A porism for triangles: $\ell$ is the pole of $P_{1} . P_{2}$ and $P_{3}$ on $\ell$ are conjugate points with respect to $C$.

Conversely, if we assume that $\varphi_{P_{n}} \circ \ldots \circ \varphi_{P_{1}}=$ id, there are three $n$-gons $A_{1}, \ldots, A_{n}, A_{1}^{\prime}, \ldots, A_{n}^{\prime}$ and $A_{1}^{\prime \prime}, \ldots, A_{n}^{\prime \prime}$ as in Figure 6. Then, by the Pascal Theorem applied to the hexagons $H_{1}$ and $H_{2}$ mentioned above, it follows that $P_{n-1}$ and $P_{n}$ must lie on the common Pascal line $\ell$ of the two hexagons. The lines $A_{1} A_{n-1}^{\prime}$ and $A_{1}^{\prime} A_{n-1}$ determine the point $X$ on $\ell$, and the lines $A_{1} A_{n-1}^{\prime \prime}$ and $A_{1}^{\prime \prime} A_{n-1}$ determine the point $Y \neq X$ on $\ell$. Thus, $\ell$ is determined by $P_{1}, \ldots, P_{n-2}$ by the construction above. Hence $P_{n-1}$ must be chosen on $\ell$ and once $P_{n-1}$ is fixed, the location of $P_{n}$ on $\ell$ follows.

Notice that the construction of the points $P_{n-1}$ and $P_{n}$ can be carried out with ruler alone as shown in the proof.

For $n=3$ we obtain the nice porism in Figure 7. In this case, $P_{1}$ is given, and $\ell$ is the polar line of $P_{1}$ with respect to $C . P_{2}$ is chosen freely on $\ell \backslash C$ and then $P_{3}$ is the conjugate of $P_{2}$ on $\ell$ with respect to $C$. This can be seen when the red or blue triangle in $C$ collapses to the line $P_{1} P_{2}$ or $P_{1} P_{3}$. For $n=4$ Theorem 10 coincides with Theorem 4.

We close this section with an example of a porism for heptagons in Figure 8.

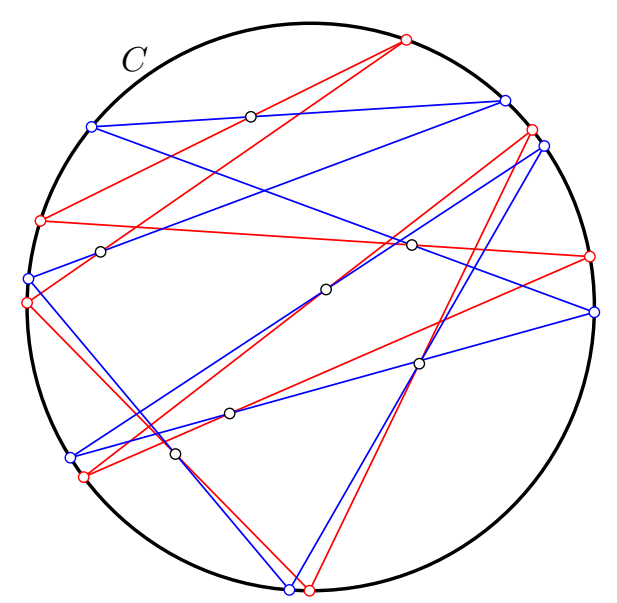

Figure 8. A porism for heptagons: The red or blue heptagon can start in any point of $C$. 


\section{Projective aspects of the Butterfly Porism}

The closing condition (1) allows a nice conclusion:

Corollary 11. If the points $P_{1}, \ldots, P_{4}$ on the line $\ell$ have the closing property with respect to a conic $C$ and if $\ell$ intersects $C$ in two points then the conjugate points $Q_{1}, \ldots, Q_{4}$ have also the closing property with respect to $C$.

Proof. The cross ratio of four points equals the cross ratio of the corresponding polar lines, i.e., we have

$$
\begin{aligned}
& \operatorname{cr}\left(R, S ; Q_{1}, Q_{4}\right)=\operatorname{cr}\left(r, s ; p_{1}, p_{4}\right)=\operatorname{cr}\left(R, S ; P_{1}, P_{4}\right)= \\
& \operatorname{cr}\left(R, S ; P_{2}, P_{3}\right)=\operatorname{cr}\left(r, s ; p_{2}, p_{3}\right)=\operatorname{cr}\left(R, S ; Q_{2}, Q_{3}\right) .
\end{aligned}
$$

See Figure 9.

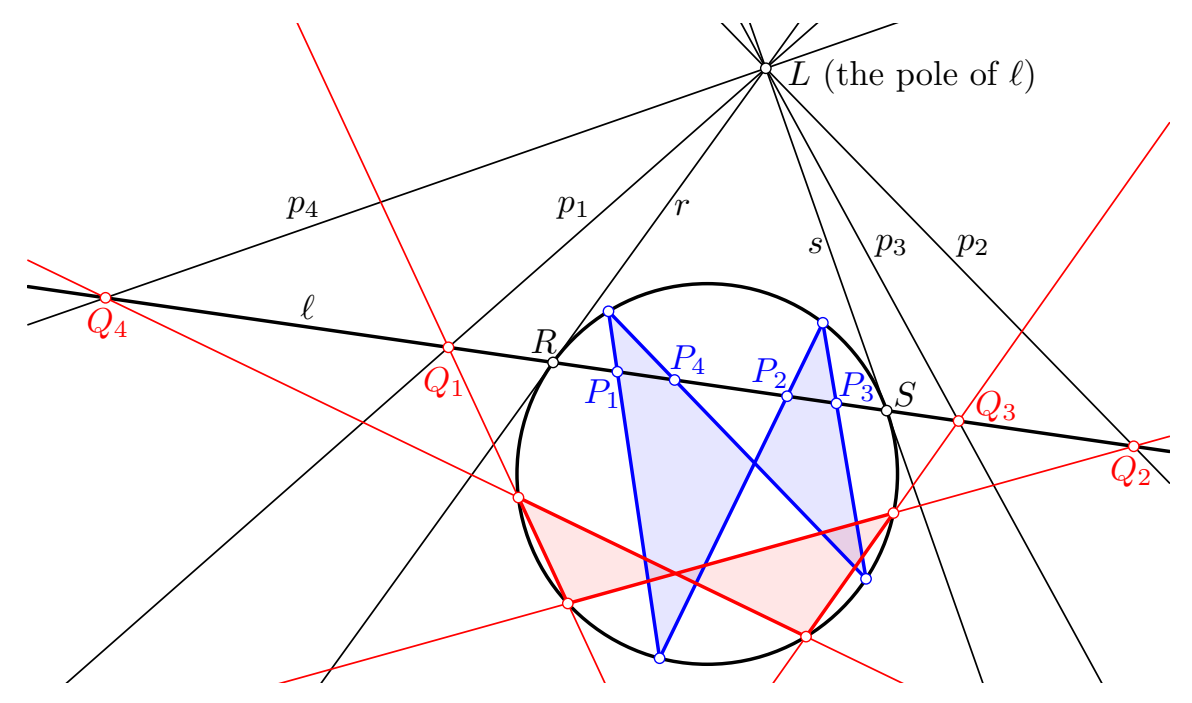

Figure 9. The closing property of the conjugate points.

Recall that Kocik [6] proved the Butterfly Porism 4 also for an arbitrary even number of collinear points $P_{1}, P_{2}, \ldots, P_{2 n}, n \geq 1$. (It follows easily from Lemma 5 that the theorem cannot hold for an odd number of points.) The previous corollary also holds in this case:

Corollary 12. If the points $P_{1}, \ldots, P_{2 n}$ on the line $\ell$ have the closing property with respect to a conic $C$ and if $\ell$ intersects $C$ in two points then the conjugate points $Q_{1}, \ldots, Q_{2 n}$ have also the closing property with respect to $C$.

Proof. For $n=1$ the assertion is trivial and the case $n=2$ is Corollary 11. We proceed by induction and suppose that we have proven the claim for some $n \geq 2$. Let us assume that the points $P_{1}, P_{2}, \ldots, P_{2(n+1)}$ have the closing property with respect to $C$, i.e.,

By Lemma 5 we have that

$$
\varphi_{P_{2(n+1)}} \circ \varphi_{P_{2 n+1}} \circ \ldots \circ \varphi_{P_{1}}=\mathrm{id} .
$$

for a point $P$ on the line $\ell$, and by Corollary 11

$$
\varphi_{P_{2(n+1)}} \circ \varphi_{P_{2 n+1}} \circ \varphi_{P_{2 n}}=\varphi_{P}
$$

$$
\varphi_{Q_{2(n+1)}} \circ \varphi_{Q_{2 n+1}} \circ \varphi_{Q_{2 n}}=\varphi_{Q}
$$

for the conjugate point $Q$ of $P$. Thus, we have

and by the induction hypothesis

$$
\varphi_{P} \circ \varphi_{P_{2 n-1}} \circ \ldots \circ \varphi_{P_{1}}=\mathrm{id}
$$

$$
\varphi_{Q} \circ \varphi_{Q_{2 n-1}} \circ \ldots \circ \varphi_{Q_{1}}=\mathrm{id} .
$$

The claim follows when we replace $\varphi_{Q}$ in (7) by (6). 
If the line $\ell$ is tangent to $C$, then the conjugate points $Q_{i}$ coincide with the point of contact. However, we can extend the above result to the case when $\ell$ does not meet $C$ :

Theorem 13. If the points $P_{1}, \ldots, P_{2 n}$ on the line $\ell$ have the closing property with respect to a conic $C$ and if $\ell$ does not meet $C$, then the conjugate points $Q_{1}, \ldots, Q_{2 n}$ have also the closing property with respect to $C$.

Proof. By applying a projective map we may assume that $C$ is the conic given by $\langle X, X\rangle=0$ and $\ell$ the line given by $\langle X, L\rangle=0$ with $L=(0,0,1)$. The projective map $\psi:\left(x_{1}, x_{2}, x_{3}\right) \mapsto\left(-x_{2}, x_{1}, x_{3}\right)$ maps $C$ to $C$, and points on $\ell$ to the conjugate points with respect to $C$. Thus, every closed polygon on $C$ with sides running successively through the points $P_{1}, \ldots, P_{2 n}$ is mapped by $\psi$ to a closed polygon on $C$ with sides running successively through the conjugate points $Q_{1}, \ldots, Q_{2 n}$.

Izmestiev noted, that since the cross ratio is invariant under projective transformations, the closing condition (1) holds for an arbitrary non-degenerate conic $C$. I.e., if $P_{1}, \ldots, P_{4}$ on a line $\ell$ have the closing property with respect to a conic $C$ they have also the closing property with respect to any other conic $D$ which intersects $\ell$ in the same points $R$ and $S$ as $C$. The reasoning is as follows: There exists a projective map $\psi$ which maps $D$ to $C$ and which has the fixed points $R$ and $S$. Let $Q_{i}=\psi\left(P_{i}\right)$ for $i=1, \ldots, 4$. Then the points $Q_{i}$ have the closing property with respect to $C$ iff $\operatorname{cr}\left(R, S ; Q_{1}, Q_{4}\right)=\operatorname{cr}\left(R, S ; Q_{2}, Q_{3}\right)$ which is equivalent to the condition $\operatorname{cr}\left(R, S ; P_{1}, P_{4}\right)=\operatorname{cr}\left(R, S ; P_{2}, P_{3}\right)$. If it is satisfied, then any closed quadrilateral on $C$ with sides running successively through the points $Q_{1}, \ldots, Q_{4}$ is mapped by $\psi^{-1}$ to a closed quadrilateral on $D$ with sides running successively through the points $P_{1}, \ldots, P_{4}$. Notice however, that in general there is no projective map which maps a closed polygon on $C$ with sides running through the points $P_{1}, \ldots, P_{4}$ to a closed polygon on $D$ with sides running through the points $P_{1}, \ldots, P_{4}$. This is due to the fact that the points $P_{1}, \ldots, P_{4}$ may be inner points of $C$ but exterior points of $D$.

Even more generally, one can replace $R$ by a point $R^{\prime}$ on $\ell$ and then determine the unique point $S^{\prime}$ such that $\operatorname{cr}\left(R^{\prime}, S^{\prime} ; P_{1}, P_{4}\right)=\operatorname{cr}\left(R^{\prime}, S^{\prime} ; P_{2}, P_{3}\right)$. Then the $P_{1}, \ldots, P_{4}$ have the closing property with respect to any conic $D$ running through $R^{\prime}$ and $S^{\prime}$.

We can generalise the observation above from four to an arbitrary even number of points:

Proposition 14. If the points $P_{1}, \ldots, P_{2 n}$ on the line $\ell$ have the closing property with respect to a conic $C$ and if $\ell$ intersects $C$ in two points $R$ and $S$, then $P_{1}, \ldots, P_{2 n}$ have the closing property with respect to any other conic $D$ through the points $R$ and $S$.

Proof. For $n=1$ and $n=2$ there is nothing more to prove. So we can proceed by induction and assume that we have proven the claim for some $n \geq 2$. Suppose that the points $P_{2(n+1)}, P_{2 n+1}, \ldots, P_{1}$ on the line $\ell$ have the closing property with respect to a conic $C$. I.e., we have

$$
\varphi_{P_{2(n+1)}}^{C} \circ \varphi_{P_{2 n+1}}^{C} \circ \ldots \circ \varphi_{P_{1}}^{C}=\text { id }
$$

where $\varphi_{P_{i}}^{C}$ means the reversion map with respect to $C$. Then, by the result for $n=2$ we have

$$
\varphi_{P_{2(n+1)}}^{C} \circ \varphi_{P_{2 n+1}}^{C} \circ \varphi_{P_{2 n}}^{C}=\varphi_{P}^{C}
$$

for some $P$ on $\ell$ and also

$$
\varphi_{P_{2(n+1)}}^{D} \circ \varphi_{P_{2 n+1}}^{D} \circ \varphi_{P_{2 n}}^{D}=\varphi_{P}^{D}
$$

for another conic $D$ through the points $R$ and $S$. So, we have

$$
\varphi_{P}^{C} \circ \varphi_{P_{2 n-1}}^{C} \circ \ldots \circ \varphi_{P_{1}}^{C}=\mathrm{id}
$$

and by the induction hypothesis

$$
\varphi_{P}^{D} \circ \varphi_{P_{2 n-1}}^{D} \circ \ldots \circ \varphi_{P_{1}}^{D}=\mathrm{id} .
$$

If we replace $\varphi_{P}^{D}$ in (9) by (8) the claim follows.

In the case when $\ell$ is tangent to $C$ in the point $R$, Izmestiev gave the closing criterion

$$
\frac{1}{R-P_{1}}-\frac{1}{R-P_{4}}=\frac{1}{R-P_{2}}-\frac{1}{R-P_{3}}
$$




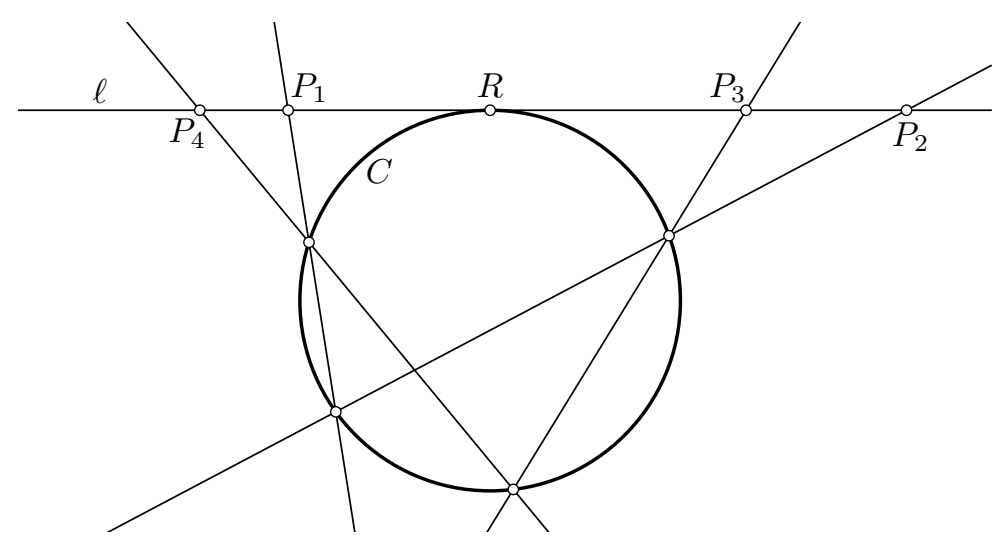

Figure 10. The case when $\ell$ is tangent to $C$.

for which it is not directly clear that it is projectively invariant. See Figure 10. The point is, that (10) can easily be reformulated as

$$
\operatorname{cr}\left(R, P_{3} ; P_{1}, P_{4}\right)=\operatorname{cr}\left(R, P_{1} ; P_{3}, P_{2}\right)
$$

which is obviously a projectively invariant condition. With the same reasoning as above, we get:

Proposition 15. If the points $P_{1}, \ldots, P_{2 n}$ on the line $\ell$ have the closing property with respect to a conic $C$ and if $\ell$ is tangent to $C$ in the point $R$, then $P_{1}, \ldots, P_{2 n}$ have the closing property with respect to any other conic $D$ which is tangent to $\ell$ in $R$.

In the case, when $\ell$ does not meet $C$ we have the following:

Theorem 16. If the points $P_{1}, \ldots, P_{2 n}$ on the line $\ell$ have the closing property with respect to a conic $C$ and if $\ell$ does not meet $C$, then $P_{1}, \ldots, P_{2 n}$ have the closing property with respect to any other conic $D$ from the three-dimensional bundle of conics which have the same complex intersections with $\ell$ as $C$.

Proof. As before it suffices to show the claim for $n=2$ to start a proof by induction. We may again assume that $C$ is given by $\langle X, X\rangle=0$ and the line $\ell$ is given by $\langle X, L\rangle=0$ with $L=(0,0,1)^{\top}$. Suppose that the points $P_{1}, \ldots, P_{4}$ on $\ell$ satisfy the closing condition with respect to $C$. It is clear that the points $P_{1}, \ldots, P_{4}$ satisfy the closing condition with respect to each member of the three-dimensional bundle $\mathcal{B}$ of conics

$$
\left\langle\left(\begin{array}{c}
x_{1}-a_{1} x_{3} \\
x_{2}-a_{2} x_{3} \\
r x_{3}
\end{array}\right),\left(\begin{array}{c}
x_{1}-a_{1} x_{3} \\
x_{2}-a_{2} x_{3} \\
r x_{3}
\end{array}\right)\right\rangle=0 .
$$

Each of these conics intersects $\ell$ in the same points $(1, \pm i, 0)$ as $C$. On the other hand, the bundle $\mathcal{C}$ of conics through $(1, \pm i, 0)$ is precisely the bundle $\mathcal{B}$.

In particular, for the case of a circle $C$ we obtain:

Corollary 17. Let $C$ and $D$ be circles and $\ell$ their radical axis. If the points $P_{1}, \ldots, P_{2 n}$ on the line $\ell$ have the closing property with respect to $C$, then they also have the closing property with respect to $D$ (see Figure 11).

We end this discussion with the following closing remark: The porisms which we presented are all formulated in the framework of the real projective plane. This allows to state the dual version in each case. For example, the dual version of the Butterfly Porism 4 reads as follows:

Theorem 18. Let $a_{1}, a_{2}, a_{3}, a_{4}$ be a quadrilateral circumscribed to a conic $C$ with sides $a_{i}$, and let $L$ be a point in $\mathbb{R P}^{2}$. Let $p_{i}$ be the line through $L$ and the intersection of the sides $a_{i}$ and $a_{i+1}$ (with cyclically read indices). Then there is $a$ quadrilateral circumscribed to $C$ with sides $a_{1}^{\prime}, a_{2}^{\prime}, a_{3}^{\prime}, a_{4}^{\prime}$ such that $a_{i}^{\prime}$ and $a_{i+1}^{\prime}$ meet on $p_{i}$ for any tangent $a_{1}^{\prime}$ of $C$ (see Figure 12). 


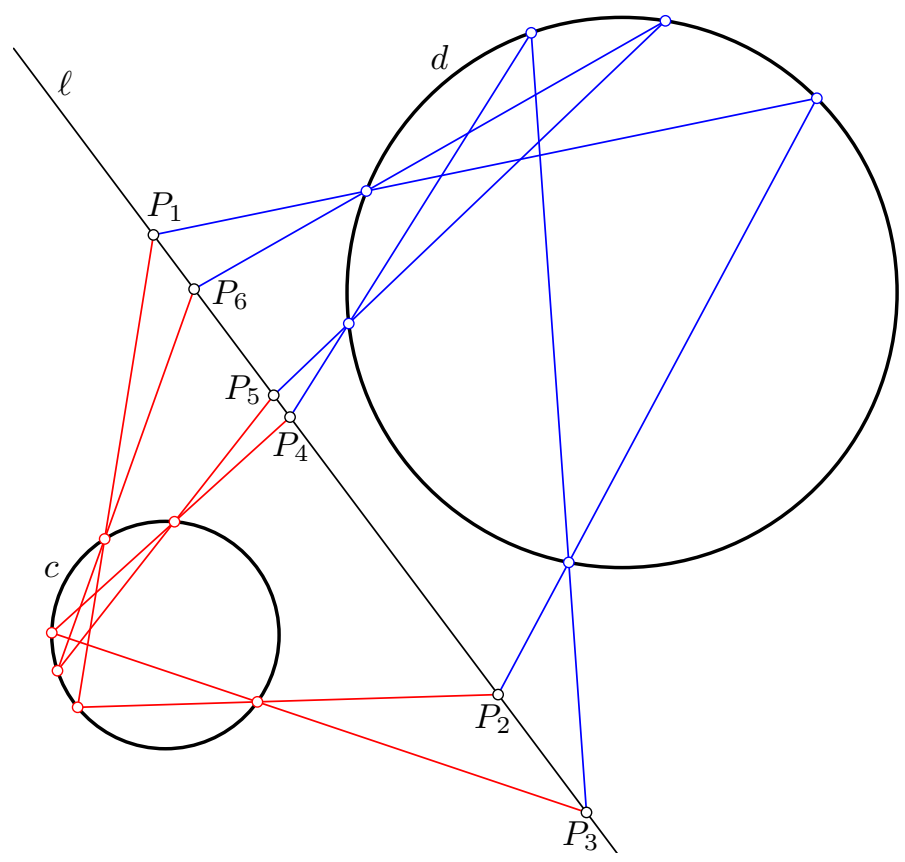

Figure 11. Closing property for points on the radical axis of two circles.

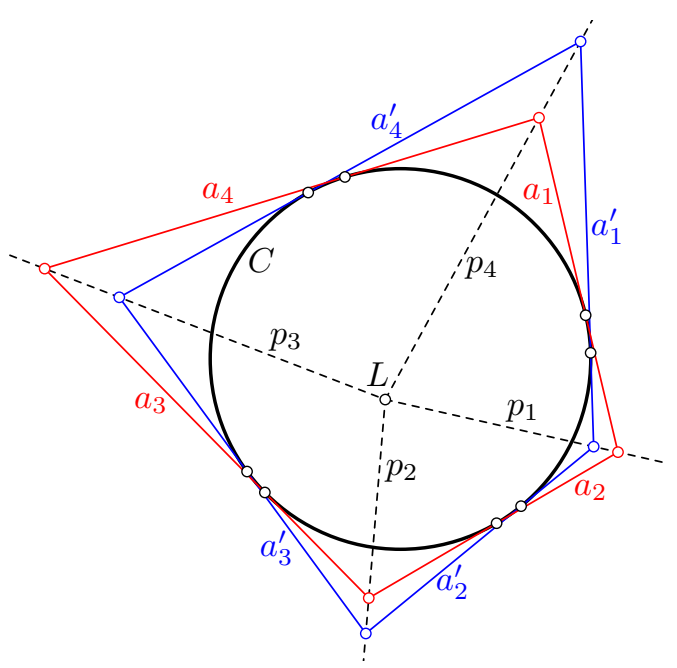

Figure 12. The dual Butterfly Theorem.

\section{References}

[1] Bogomolny, A.: The butterfly theorem. Interactive Mathematics Miscellany and Puzzles. http://www.cut-the-knot.org/pythagoras/Butterfly.shtml, accessed February 4, 2021.

[2] Coxeter, H. S. M., Greitzer, S. L.: Geometry revisited, volume 19 of New Mathematical Library. Random House, Inc. New York (1967).

[3] Izmestiev, I.: A porism for cyclic quadrilaterals, butterfly theorems, and hyperbolic geometry. Amer. Math. Monthly. 122 (5), 467-475 (2015).

[4] Jones, D.: Quadrangles, butterflies, Pascal's hexagon, and projective fixed points. Amer. Math. Monthly. 87 (3), 197-200 (1980).

[5] Klamkin, M. S.: An Extension of the Butterfly Problem. Math. Mag. 38 (4), 206-208 (1965).

[6] Kocik, J.: A porism concerning cyclic quadrilaterals. Geometry, Article ID 483727: 5 pages (2013).

[7] Sliepčević, A.: A new generalization of the butterfly theorem. J. Geom. Graph. 6 (1), 61-68 (2002).

[8] Volenec, V.: A generalization of the butterfly theorem. Math. Commun. 5 (2), 157-160 (2000). 


\section{Affiliations}

LORENZ HALBEISEN

ADDRESS: ETH Zürich, Department of Mathematics, 8092 Zürich, Switzerland.

E-MAIL: lorenz.halbeisen@math.ethz.ch

ORCID ID: 0000-0001-6078-7237

NORBERT HUNGERBÜHLER

AdDRESS: ETH Zürich, Department of Mathematics, 8092 Zürich, Switzerland.

E-MAIL: norbert.hungerbuehler@math.ethz.ch

ORCID ID: 0000-0001-6191-0022

MARCO SCHILTKNECHT

AdDRESS: ETH Zürich, Department of Mathematics, 8092 Zürich, Switzerland.

E-MAIL: marcosc@student.ethz.ch

ORCID ID: 0000-0001-7381-2999 